\title{
Base Pressure Measurements on a Circular Cylinder in Subsonic Cross Flow
}

\author{
J. R. Ackerman ${ }^{1}$, J. P. Gostelow ${ }^{2}$, A. Rona ${ }^{3}$ \\ University of Leicester, Leicester, LE1 7RH, UK \\ W. E. Carscallen ${ }^{4}$ \\ Institute for Aerospace Research, \\ National Research Council of Canada, Ottawa, K1A 0R6, Canada
}

\begin{abstract}
A circular cylinder was tested in cross flow over the subsonic speed range. Timeresolved pressure distributions give information on surface pressure fluctuations and the corresponding drag and base drag coefficients are provided. The Strouhal number variation is compared with the measurements of other authors. Flow changes at higher subsonic velocities and into the transonic range are described. At Mach numbers above 0.6 the changing strength of the vortices reduces the base drag coefficient up to a Mach number of 0.9 , where the onset of sonic flow increases the drag. Time-resolved base pressure fluctuations at low Mach numbers are in agreement with the findings of other researchers with regard to the relative time spent in vortex formation and shedding. As the Mach number increases the time spent in vortex formation becomes equal to that spent in shedding. The paper concentrates on providing detailed base pressure data rather than attempting to produce universal correlations. Physical explanations have been given, where possible, to assist toward a more general modeling of the problem.
\end{abstract}

\section{Nomenclature}

$\begin{array}{ll}C_{D} & \text { Drag coefficient } \\ C_{B D} & \text { Base drag coefficient } \\ C_{p} & \text { Static pressure coefficient } \\ M a & \text { Mach number } \\ P & \text { Total pressure }(\mathrm{Pa}) \\ R e & \text { Reynolds number } \\ S t & \text { Strouhal number } \\ f & \text { Frequency }(\mathrm{Hz}) \\ p & \text { Static pressure }(\mathrm{Pa}) \\ S & \text { Root mean square value } \\ \theta & \text { Cylinder azimuth (degrees) } \\ \text { Subscripts } \\ S & \text { Cylinder surface conditions } \\ 01 & \text { Free stream stagnation condition } \\ 02 & \text { Wake stagnation condition } \\ 180 & \text { Cylinder trailing edge } \\ \text { pla } & \text { Phase-averaged pressure coefficient data }\end{array}$

\section{Introduction}

This paper reports on an experimental investigation of the base pressure variation over the subsonic speed range on a circular cylinder in cross flow. The initial context and motivation emerged from work on a family of gas turbine nozzle vanes with a blunt trailing edge ${ }^{1}$. Work by Denton and $\mathrm{Lu}^{2}$ had established that for such blades this area of reduced static pressure around the trailing edge was a major contributor to total pressure loss. Turbine blades with a thick trailing edge, such as those investigated by Carscallen et al. ${ }^{3}$, have a high loss penalty associated with the trailing edge. This loss penalty is greater than would be expected from a simple backward-facing step and had been quantified by Deych et al. ${ }^{4}$ and Craig and Cox $^{5}$.

\footnotetext{
'Tepartment of Engineering, University of Leicester, Leicester, LE1 7RH, U.K.

${ }^{2}$ Emeritus Professor, Department of Engineering, University of Leicester, Leicester, LE1 7RH, U.K. Member AIAA.

${ }^{3}$ Lecturer, Department of Engineering, University of Leicester, Leicester, LE1 7RH, U.K. Member AIAA.

${ }^{4}$ Manager of Research and Technology, GTL, IAR, National Research Council, Ottawa, K1A 0R6, Canada. Member AIAA
} 
The high losses remained unexplained until high speed schlieren photography was applied to cascades ${ }^{6}$. In subsonic flows past a blunt trailing-edged turbine blade, or past its simplified model, a circular cylinder, periodic vortex shedding is almost always present up to a Mach number of about 1.2. This vortex shedding was found to be present in the wakes of blades with thick trailing edges, often associated with pressure waves propagating upstream along the blade surfaces when the local free stream flow was subsonic. These appeared at the vortex shedding frequency. The unexplained losses were clearly associated with the vortex shedding process. Hoerner ${ }^{7}$ had earlier correlated base drag for airfoils and backward-facing steps; he found that the base pressure of an airfoil was consistently lower than that of an equivalent backward-facing step. He attributed this discrepancy to the existence of a von Kármán vortex street in the wake of the airfoil. For turbine blades, Cicatelli and Sieverding ${ }^{8}$ conducted an investigation into the effect of vortex shedding on the base region flow. They found that the pressure in this region fluctuated by as much as $8 \%$ of the downstream dynamic head near separation and by $4.8 \%$ in the base region. This would seem to indicate that the instantaneous base pressure could be significantly different from the time averaged value; this gave poor computational results if steady state methods, such as conventional RANS, were used. It has been concluded" that, for bluff body flows, "calculation methods which neglect base pressure effects are incapable of accurately calculating the flow patterns or the total pressure loss".

Despite the importance of trailing edge vortex shedding for turbine blading, this problem is more widespread and generic in nature. It was considered important to reduce the study to the most significant variables and to attempt to address the problem in a relatively generic form. For this reason it was decided to simplify the geometry and boundary conditions to an isolated and rigidly mounted circular cylinder in subsonic cross flow. Unlike the case of a turbine blade, the time-averaged boundary layers on either side of the circular cylinder are symmetrical and sufficiently thin not to smear the shed vortices.

The base pressure is known to be strongly dependent on Mach number. At supersonic speeds, the main cause of low base pressure is the strong spatial variations of pressure through shocks and expansions; this tends to be a relatively steady process. At subsonic speeds, shocks only play a role as the velocity reaches critical levels and, in general, the unsteady process of vortex shedding is more important. These are therefore two quite distinct compressibility effects. This investigation focuses exclusively on the subsonic speed range.

The classical von Kármán vortex street is the most usual shedding mode at subsonic speeds and results in predictable configuration and frequency characteristics. These vary with the thickness of the boundary layers at separation and therefore with Reynolds number. In the current investigation, wake traverses of total pressure and temperature were taken and will be the subject of a further publication. In addition to the wake traverses, surface pressure readings from around the cylinder were also taken in an effort to increase the understanding of the relationship between vortex shedding and the base pressure of cylinders and turbine blades.

Limited data on circular cylinders do exist and the purpose of this paper is to extend that rather sparse data set. Systematic modeling is ultimately required, however, and calls for a greater physical understanding of the rather complex phenomena of vortex shedding and the base region than we presently have.

\section{Facility and Instrumentation for Circular Cylinder Testing}

The National Research Council (NRC) $1.5 \mathrm{~m}$ trisonic blowdown wind tunnel in Ottawa was used for this investigation with a two-dimensional (2D) flow working section fitted. The test section measured $0.381 \mathrm{~m} \times$ $1.524 \mathrm{~m} \times 3.581 \mathrm{~m}$. The air supply comprised three $143 \mathrm{~m}^{3}$ tanks that could be pressurized up to $2.13 \mathrm{MPa}$ (21 atm). In this mode, the wind tunnel had a discharge Mach number range of 0.1 to 0.9 and was run at a constant Reynolds number of $6.83( \pm 0.03) \times 10^{5}$. The Reynolds number is based on cylinder diameter and free stream inflow velocity. The operating Reynolds number range and run time were dependent on Mach number and set by adjusting the total to static pressure ratio of the test section. Blowdown operation resulted in the free stream total temperature dropping over the course of each run as the air in the supply tanks expanded. The model was mounted $2.388 \mathrm{~m}$ downstream of the end of the contraction on reinforced mounts in a threecomponent balance. Due to the method of reinforcement, it was impossible to take measurements of the forces acting on the cylinder from the balance. Tunnel wall boundary layer control was applied using ejectoraugmented sidewall suction through $0.602 \mathrm{~m} \times 0.452 \mathrm{~m}$ porous plates. The balance could be rotated through a range of $\pm 55^{\circ}$ at a rate of $15^{\circ}$ per second.

Two series of tests were conducted, in 2000 and 2002 respectively, and the results of surface pressure measurements are designated accordingly. The model was an aluminum circular cylinder with a diameter of $37.26 \mathrm{~mm}$ and a span of $381 \mathrm{~mm}$. In the 2000 series, a single 0.062 inch diameter Kulite XCQ-062-25D ultraminiature pressure transducer was mounted so that the B-screen, which covered the pressure sensitive diaphragm, was flush with the cylinder surface at midspan. For the 2002 series, four 0.093 inch diameter Entran transducers were mounted equally spaced around the circumference. 


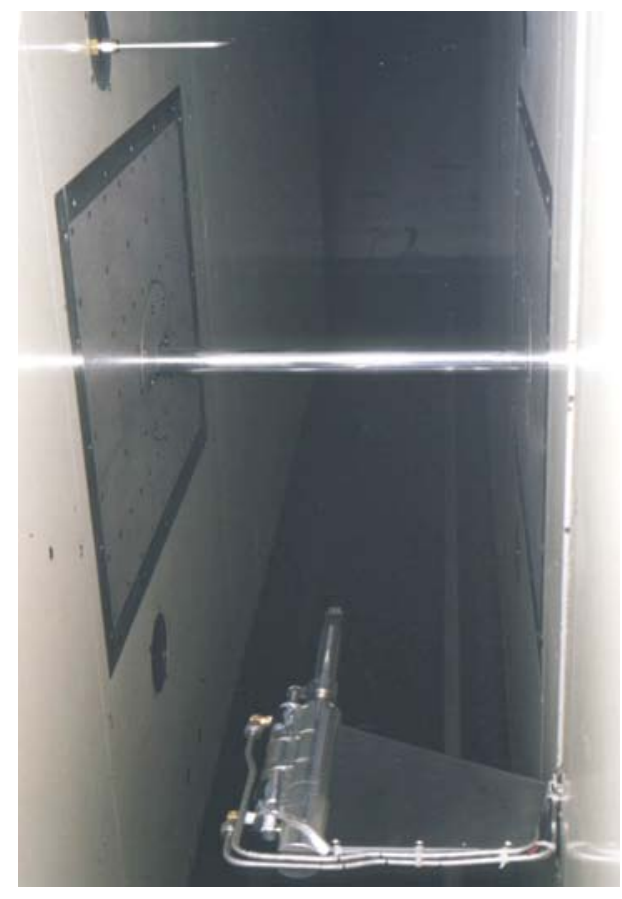

\section{Figure 1. Cylinder and wake probe mounted in wind tunnel}

A wake probe used for the traverse downstream of the cylinder consisted of a total temperature probe, developed at Oxford University by Buttsworth and Jones ${ }^{10}$, alongside a tubemounted Kulite XCQ-062-25D ultraminiature pressure transducer. These probes were mounted in a glass holder attached to the end of a stainless steel tube. The Kulite pressure transducer was mounted so that its face was looking directly into the flow, allowing it to measure the total pressure. The probes were mounted so that their tips were all level and aligned along the horizontal spanwise tunnel axis.

The vortex shedding was assumed to be two-dimensional, thus mounting the probes in this way ensured that they would all see the vortex passing events simultaneously. The probe sting was mounted for wake traverse using a triangulated support. The sting and support were designed so that the probe tips would be six cylinder diameters or $223.6 \mathrm{~mm}$ downstream of the model's leeward-most point, as shown in Fig. 1.

Surface pressure measurements were taken in $5^{\circ}$ increments from $10^{\circ}$ below the leading edge through to $10^{\circ}$ below the trailing edge. The forward stagnation point is the zero location and the angular orientation is clockwise. The cylinder could be rotationally indexed, thus minimizing the number of surface pressure transducers required. Data were taken for two seconds at each position. The traverse was taken over two blowdowns. In the first of these the cylinder was traversed from $10^{\circ}$ below the leading edge around to $100^{\circ}$ above it. The second traverse covered the $80^{\circ}$ to $190^{\circ}$ locations. Alongside each surface pressure reading, a two-second record was taken from the wake probe pressure transducer that acted as a phase reference. Phase reference locations were chosen for pressure measurements with strong signals that were dominated by the vortex shedding fundamental frequency. The signals were low-pass filtered at $40 \mathrm{kHz}$, for anti-aliasing, before being recorded at a sample rate of $100 \mathrm{kHz}$ directly onto the PC through National Instruments data acquisition cards.

\section{Results and Discussion}

Figures 2 and 3 show time-averaged surface pressure distributions for the circular cylinders used from the 2000 and 2002 series of tests respectively. The surface pressures are presented in the form of pressure coefficient:

$$
C p_{t}=\frac{p_{s t}-p_{1}}{P_{T 1}-p_{1}}
$$

Superimposed on the mean values, $\langle C p\rangle_{t}$, are the maximum and minimum levels of raw pressure coefficient, $C p$, and the root mean square (RMS) of the raw and phase lock averaged fluctuations in $C p$. The RMS of the pressure coefficient for both raw and phase lock-averaged data is given in Eq. 2:

$$
s_{X}=\sqrt{\frac{1}{N-1} \sum_{i=1}^{N}\left(X-\langle X\rangle_{t}\right)^{2}} ;
$$

where $X$ is the quantity being examined.

The 2000 series and 2002 series results in Figs. 2 and 3 show that the pressure distributions at Mach numbers of 0.5 and 0.7 are broadly similar; however at Mach 0.6 there are considerable differences. This can best be quantified by finding the coefficients of form drag, $C_{D}$, and base drag, $C_{B D}$, defined in Eqs. 3 and 4, for the various profiles and plotting them against Mach number, in Fig. 4.

$$
C_{D}=\int_{\theta=180}^{\theta=0} C p d(\sin \theta)
$$




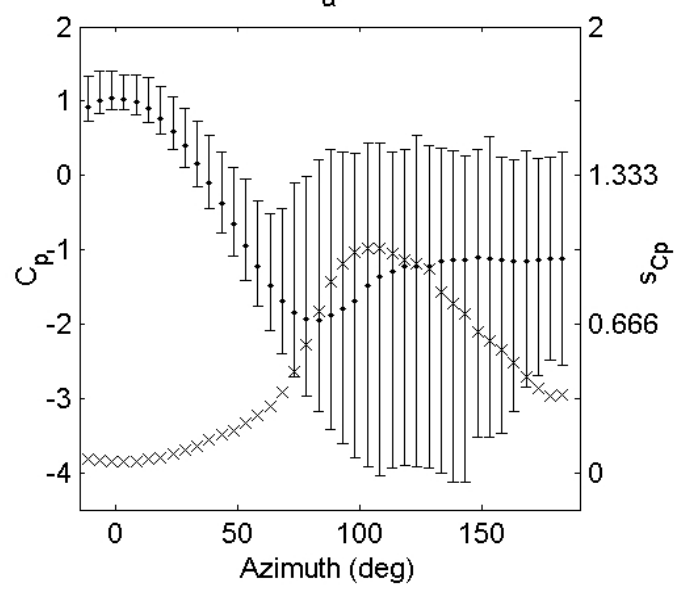

c

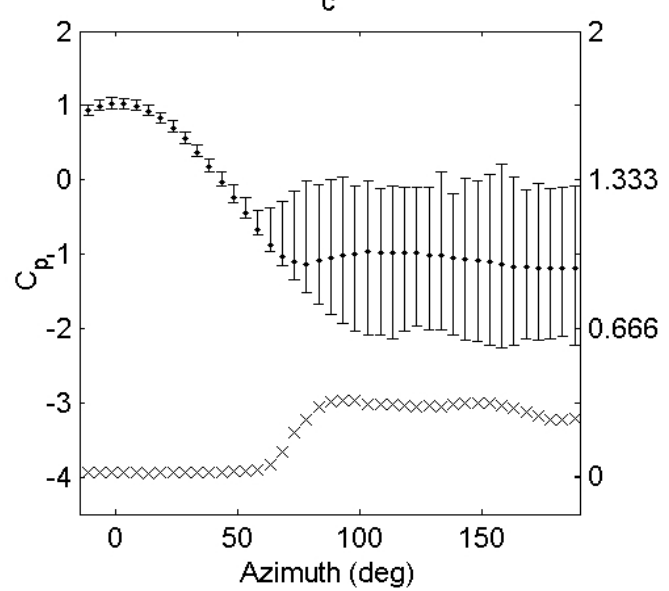

e

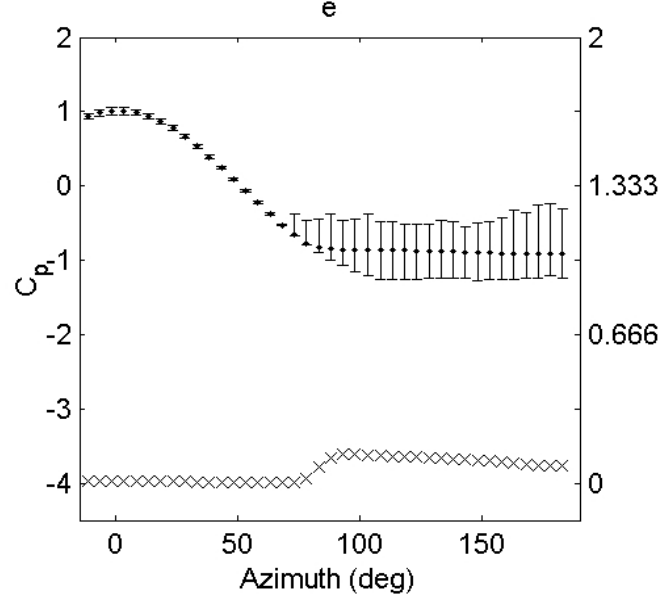

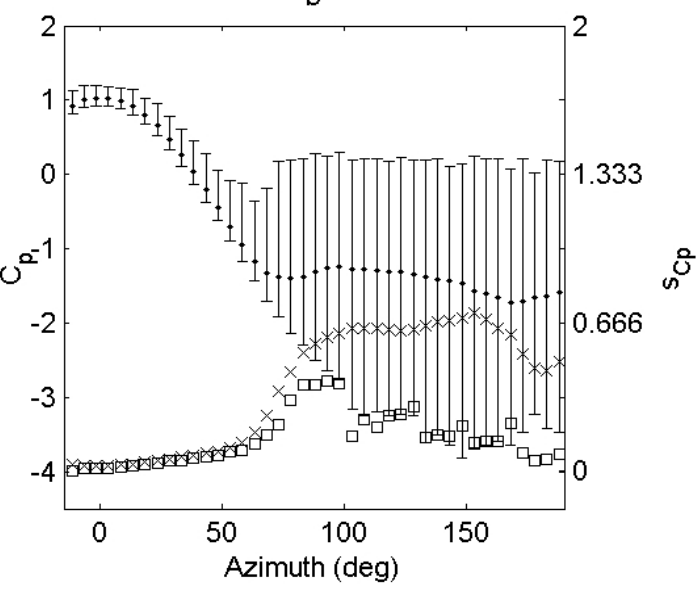
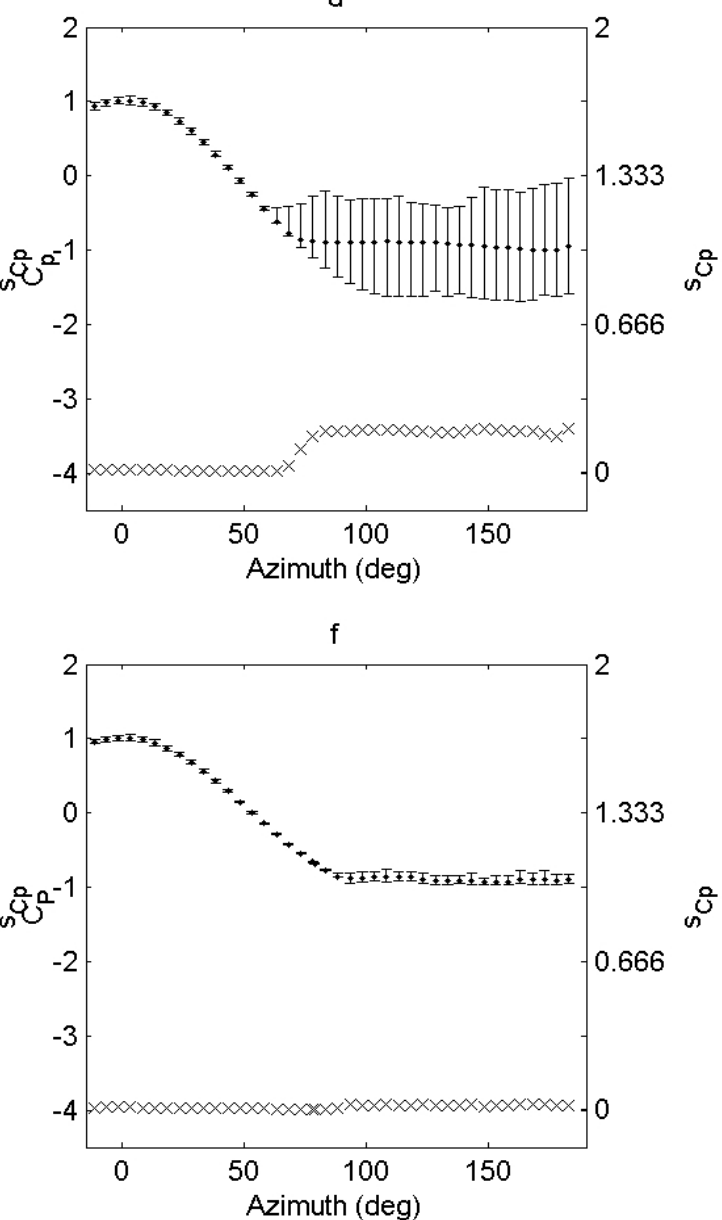

Figure 2. Pressure distributions from the 2000 series data

a) $\mathrm{Ma}=0.5$, b) $\mathrm{Ma}=0.6$, c) $\mathrm{Ma}=0.7$, d) $\mathrm{Ma}=0.8$, e) $\mathrm{Ma}=0.9$, f) $\mathrm{Ma}=0.95$

Symbols: $\cdot\left\langle C_{P_{p}}\right\rangle_{t}, \times s_{C p_{-} \text {raw }}, \square s_{C p \_p l a}$

Error bars indicate maximum and minimum levels of fluctuation. 

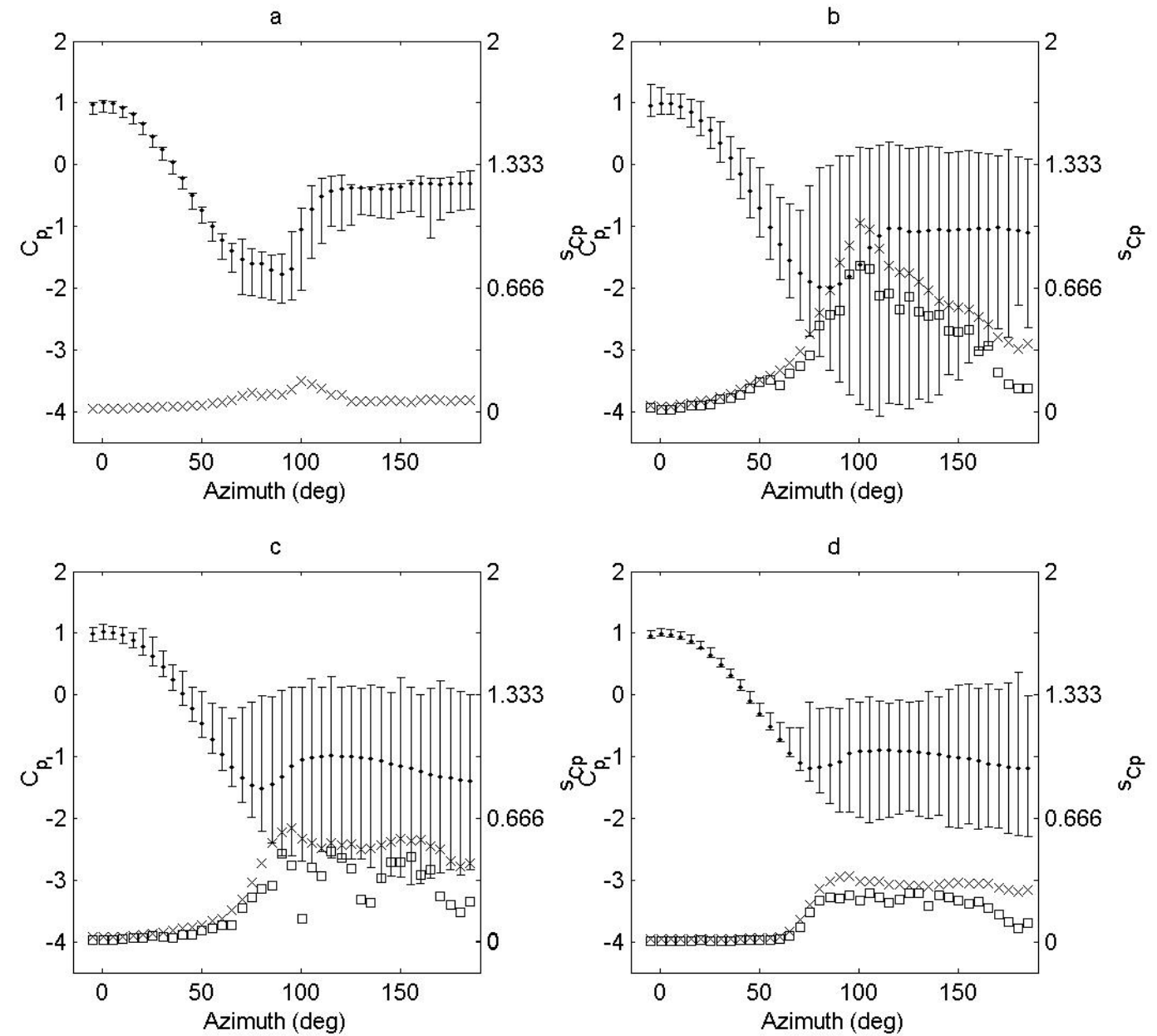

Figure 3. Pressure distributions from the 2002 series data

a) $\mathrm{Ma}=0.4$, b) $\mathrm{Ma}=0.5$, c) $\mathrm{Ma}=0.6$, d) $\mathrm{Ma}=0.7 \quad$ Symbols: $\cdot\left\langle C_{P}\right\rangle_{t}, \times s_{C p_{-} r a w}, \square s_{C p_{-} p l a}$, Error bars indicate maximum and minimum levels of fluctuation.

$$
C_{B D}=\int_{\theta=180}^{\theta=s e p} C p d(\sin \theta)
$$

where sep indicates the time-averaged location where the boundary layer separates from the leeward cylindrical surface.

This method of calculating profile drag only includes contributions from form drag and neglects skin friction and drag created by the loss of total pressure across local shock waves emanating from the cylinder surface. At a Reynolds number of $6.8 \times 10^{5}$ the contribution to profile drag from skin friction drag is very small ${ }^{11}$. Given that the flow is transonic, the contribution to drag by total pressure loss through shocks will be small although it will increase, along with the discrepancy caused by neglecting it, as the Mach number approaches unity.

Before considering the differences between the two sets of data, an explanation of the changes in $C_{D}$ over the Mach number range will give an insight into the reason for the differences. From Fig. 4 it can be seen that both $C_{D}$ and $C_{B D}$ start low and then rise to a maximum at Mach 0.6, before falling off at Mach 0.7 and then rising again slowly at the higher Mach numbers. These variations over the Mach number range are compatible with the compressible flow regimes described by Zdravkovich ${ }^{11}$, namely, shockless, intermittent shock wave, permanent shock wave and wake shock wave. Much of the following discussion draws upon that work.

At Mach 0.4, the flow is in the shockless regime and so is strongly Reynolds number dependent; it is this that determines $C_{D}$. In this case, given the Reynolds number used in the test, the flow falls into the Transitional Boundary Layer 3 (TrBL3) subsonic regime ${ }^{11}$. This explains the lack of vortex shedding and low $C_{D}$ observed 


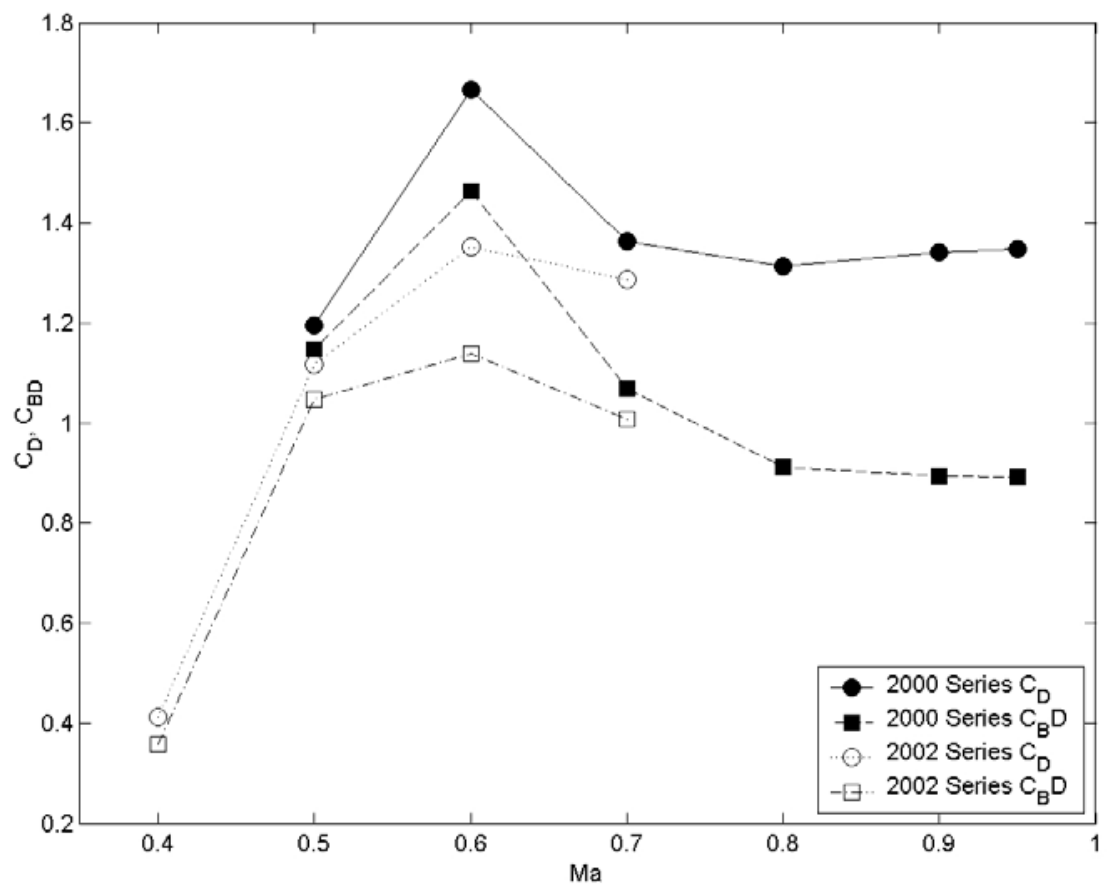

Figure 4. Drag and base drag coefficients over the Mach number range $0.4 \leq \mathrm{Ma} \leq 0.95$

at this Mach number, as shown by the narrow error band and low $s_{\text {Cp_raw }}$ in Fig. 3a compared to Fig. 3b-d: "Further increase in $\operatorname{Re}$ [into the $\mathrm{TrBl} 3$ regime] brings transition to the primary laminar separation line in an irregular manner. This leads to the disruption and fragmentation of separation bubbles along the span of the cylinder. The irregularly fragmented separation line prevents eddy [vortex] separation..." The lack of vortex shedding permits the high level of pressure recovery, seen in Fig. 3a, which results in the low $C_{D}$.

Just above Mach 0.4 the flow becomes critical, i.e. local regions of flow about the cylinder become supersonic and the flow enters the intermittent shock wave regime. The oscillating flow field present in this regime, which results in the flow being supersonic only on one side of the cylinder at a time, leads to increased pressure fluctuations compared to the previous Mach number. This can be clearly seen in the change in RMS level and maximum and minimum pressure levels in Figs. $3 \mathrm{a}$ and $3 \mathrm{~b}$, where the free stream Mach numbers are 0.4 and 0.5 respectively.

As the free stream Mach number increases beyond the critical limit, the region behind the cylinder in which the vortices are formed shortens. The increase in $C_{D}$ leading up to Mach 0.6 is a result of the commencement of vortex shedding, the shortening of the vortex shedding region with increasing Mach number and the formation of the local shock waves as the intermittent shock wave regime strengthens. Beyond around Mach 0.65 , the flow enters the permanent shock wave regime. The permanent shock wave regime causes the movement of the location of formation and shedding of the vortices downstream of the cylinder surface. This lengthens the formation region, increases the pressure recovery and gives a slightly earlier separation. This in turn leads to the reduction in $C_{D}$ at Mach 0.7 .

Once the flow enters the wake shock wave regime, at just below Mach 0.8, the vortex formation region becomes elongated; a normal shock grows at the point of vortex roll up and the drag on the cylinder increases. This increase can be seen in Fig. 4. As the flow moves further into the regime at Mach 0.95, $C_{D}$ again increases slightly and no vortex shedding is detected on the surface of the cylinder.

Figure 4 also highlights the discrepancy in $C_{D}$ between the two sets of results at Mach 0.6. Below and above Mach 0.6 the differences in $C_{D}$ between the two sets of results are 0.08 at Mach 0.5 and 0.07 at Mach 0.7. These differences are small, approximately $7 \%$ and $5 \%$ of the overall $C_{D}$ at Mach 0.5 and 0.7 respectively and can be attributed to experimental error and the slight change in Reynolds number as the Mach number has not yet increased sufficiently to remove all Reynolds number effects. However at Mach 0.6 the difference is 0.31 , quite considerable when one remembers that at Mach $0.4 C_{D}$ is 0.41 , suggesting that there is a difference between the boundary layer flow in the 2000 and 2002 series results. To aid the investigation into the $C_{D}$ discrepancy, the 2000 series pressure profiles are shown in Fig. 5 with the 2002 series profiles superimposed, for Mach numbers of 0.5, 0.6 and 0.7 . 
a

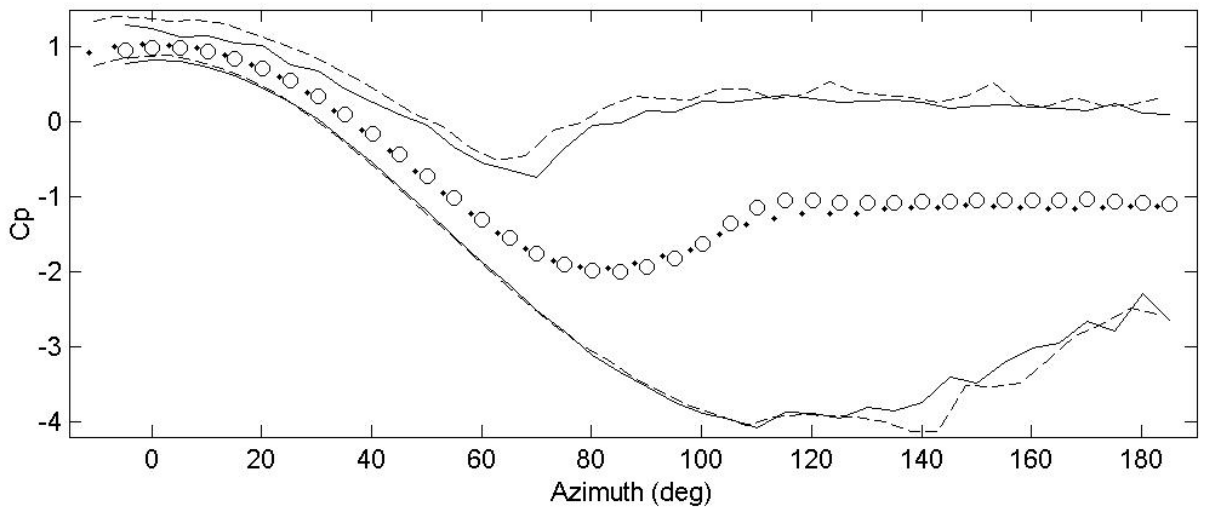

b

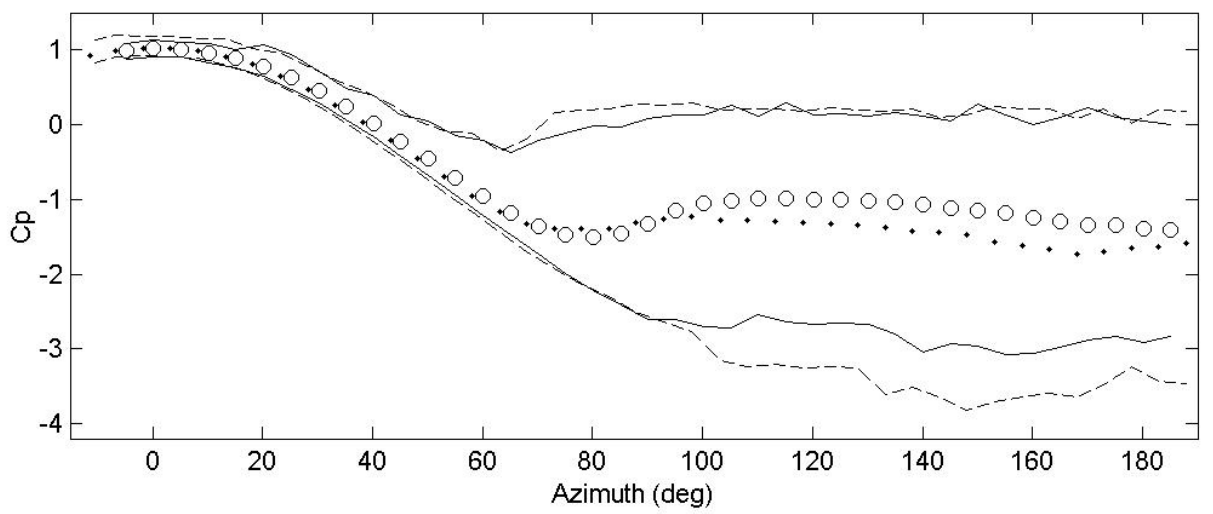

C

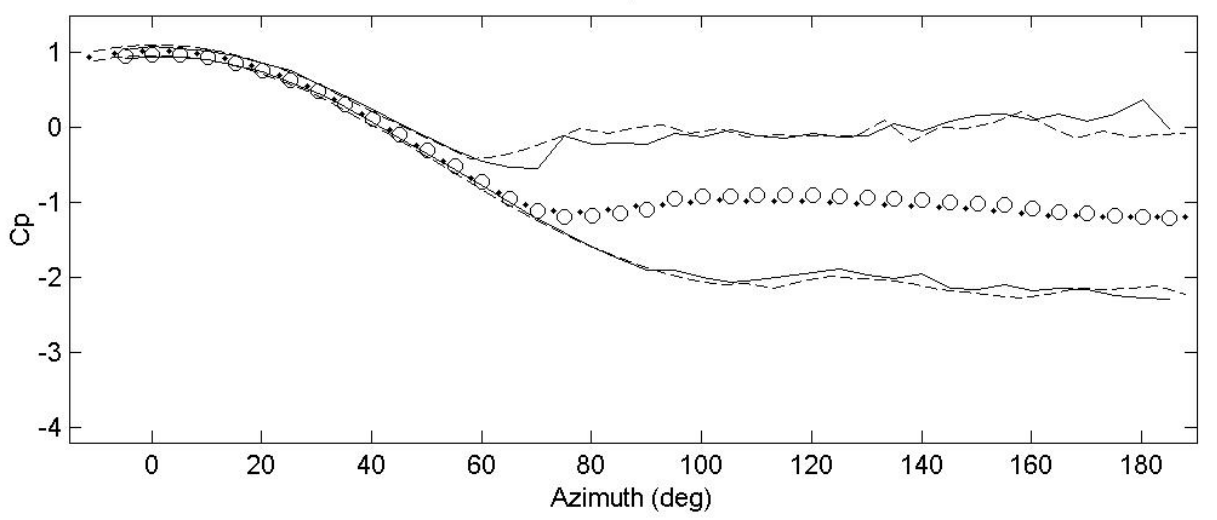

Figure 5. Pressure coefficient distributions for the 2000 series and the 2002 series tests a) Mach 0.5, b) Mach 0.6, c) Mach 0.7

Symbols: $\cdot 2000$ series $\left\langle C_{P}\right\rangle_{\text {t }}, 02002$ series $\left\langle C_{P}\right\rangle_{\text {t }}$,

Dashed line: 2000 series $\left(C_{P}\right)_{\text {Max/Min }}$, Continuous line: 2002 series $\left(C_{P}\right)_{\text {Max Min }}$

In Fig. 5a the 2002 series time-averaged surface pressure distributions, o, follow closely the 2000 series distributions from the upstream stagnation point $\left(\theta=0^{\circ}\right)$ to an azimuthal angle of about $100^{\circ}$. In the range $100^{\circ} \leq \theta \leq 130^{\circ}$ there is a greater static pressure recovery in the 2002 series data than in the 2000 series data. This difference indicates the presence of an early laminar separation in the 2000 series tests whereas the separation in the 2002 series tests is delayed by about ten degrees of azimuth. This effect is more pronounced at Mach 0.6 , where the flow separates at $\theta \approx 90^{\circ}$, causing a reduction in base pressure that affects the entire leeward face $\left(90^{\circ} \leq \theta \leq 180^{\circ}\right)$. This separation is accompanied by a larger excursion between the minimum and maximum pressure coefficients, indicated by the dashed lines. This is indicative of very strong vortex shedding. The Mach 0.7 pressure distribution also shows a greater pressure recovery at $\theta \approx 95^{\circ}$ for the 2002 series tests. 


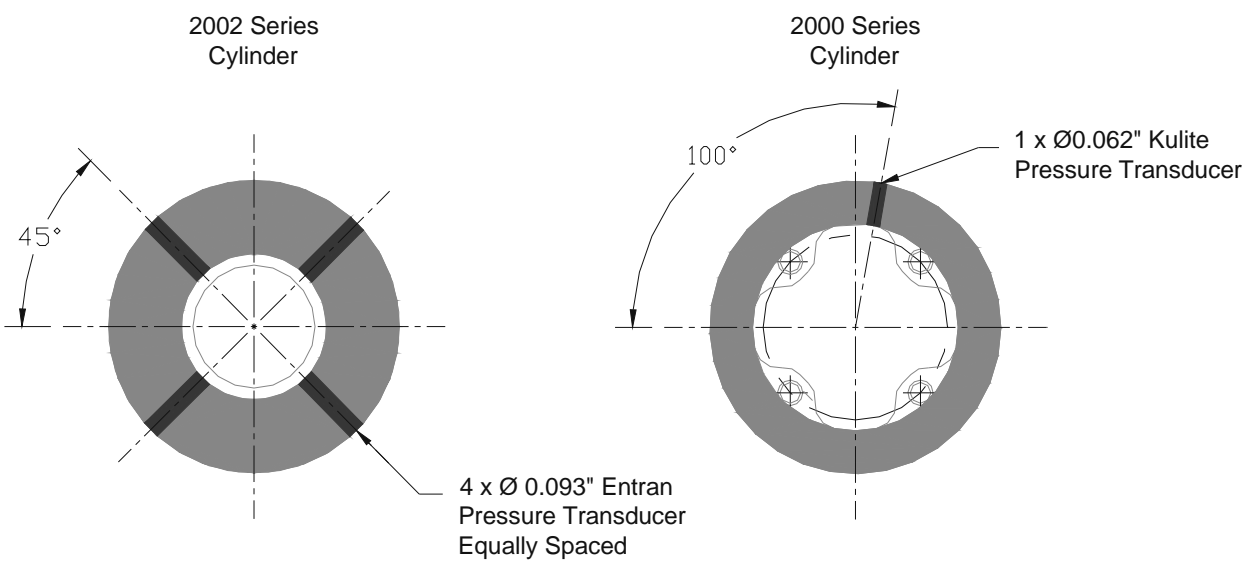

Figure 6. Location of pressure transducers within cylinder models

series tests. This shows that for all three Mach numbers the boundary layer at separation is mostly laminar whereas the cylinder boundary layer during the 2002 series tests is either turbulent or going through transition at separation. The tripping of the boundary layer into a turbulent or transitional state may have happened in one of two ways. The first possibility is that a change in the running conditions of the facility itself between the two sets prompted the change. However the free-stream Reynolds numbers for the 2000 series and 2002 series tests were virtually identical, $6.86 \times 10^{5}$ and $6.80 \times 10^{5}$ respectively. Measurements taken during the later set of tests were thought to be those of a cylinder with a tripped boundary layer. Tests of the free stream pressure fluctuations conducted on the facility indicate that the fitting of a new control valve port ring shortly after the 2000 series tests actually reduced the free-stream turbulence. This means that if the only changes between the tests were those to free-stream conditions the boundary layer should have been more laminar during the 2002 series test than in the 2000 series tests. Clearly this is not the cause of the change in separation position and pressure recovery between the two sets of data.

The second possibility is that the presence of four pressure transducers positioned around the midspan of the cylinder in the 2002 series of tests as opposed to the single transducer in the 2000 series experiments (Fig. 6) may have caused the 2002 measurements to be those of a cylinder with a tripped boundary layer. The presence of the pressure transducers on the surface of the cylinders presents flat spots, the transducer diaphragm making the surface curvature discontinuous. While this was minimized by fitting each transducer so that the face was flush with the upstream and downstream edges of the mounting hole, discontinuities were formed, with the larger transducer creating the larger discontinuities. Because of the larger diaphragm diameter, the presence of the transducers creates discontinuities in the surface curvature that are larger for the Entran transducers. By themselves, the discontinuities may or may not cause the boundary layer to become transitional or turbulent. However, when one considers that the critical Mach number for a circular cylinder, above which the flow becomes locally supersonic, is around 0.4, then at the higher Mach numbers of the 2002 series these discontinuities may well cause the creation of local shock waves which cause the boundary layer to trip. During the 2000 series of tests there was only one transducer on the cylinder surface and the discontinuity caused by it was small. This means that any alteration to the boundary layer as a result of shock waves coming from it occurred after the flow has passed over it. Since any effect this has on the flow is not easily communicated upstream due to the flow being sonic, the readings taken will effectively be those of a laminar boundary layer with shock induced laminar separation. This is not the case for the 2002 series of tests.

The windward-facing transducers on the cylinder used for the 2002 series tests ensured that the flow seen by the leeward transducers would always be tripped. Thus the leeward readings are all of a tripped boundary layer with shock induced turbulent separation.

It would seem then that the most likely cause of the differences in $C_{D}$ and $C_{B D}$ between the series of tests is caused by the presence of discontinuities in the surface curvature due to the extra transducers. While this does mean that the exact flow conditions for the two sets of results differ, it also means that data exist for vortex shedding from a circular cylinder with both a purely laminar boundary layer and a tripped turbulent or transitional boundary layer. This will be borne in mind during the subsequent discussion of the results.

Despite the differences between the drag coefficients and base drag coefficients taken from the 2000 series and 2002 series, both sets display the same trend. As the vortex shedding becomes established, at Mach 0.5 and 0.6, there is a significant rise in $C_{D}$ and $C_{B D}$ in Fig. 4. Above Mach 0.6, the coefficients fall off at Mach 0.7 and then, in the case of $C_{D}$, rise slowly; and in the case of $C_{B D}$ fall slowly. The large difference between the coefficients at Mach 0.6 and Mach 0.7 may be attributed to the change in flow regime from intermittent shock 
wave to permanent shock wave at around Mach 0.65. Given that the coefficients do not change much after Mach 0.7, it can be deduced that the change in the coefficients between Mach 0.6 and 0.7 is due to the flow around the cylinder containing supersonic regions throughout the vortex shedding cycle. The slight changes after Mach 0.7 are due to delayed separation and greater pressure recovery.

Another point of difference between the two sets of data is the RMS levels of the phase lock averaged and raw $C_{P}$ at Mach 0.6. The 2000 series results have a notable drop in the level of the RMS between the raw and phase lock averaged data that does not occur in the 2002 series data, Figs. $2 b$ and $3 c$. The cause of this is the use of wake pressure readings as a phase reference during the 2000 series results. While the wake readings do provide a reasonable phase reference, there is a greater level of jitter in the wake pressure reading than in those taken on the cylinder surface. This has the effect of slightly smearing the phase lock averaged data, resulting in a lower RMS of the fluctuations. This means that, while the 2000 series phase lock-averaged $C_{P}$ are valid data, they are not of such high quality as the 2002 data.

Another flow feature that shows up clearly in Fig. 5 is the drop in the level of pressure fluctuation on the windward side, forward of around the $60^{\circ}$ azimuth, as the Mach number increases. Inspection of Figs. 2 and 3 shows that this is generally true from Mach 0.5 upwards; it is caused by the strengthening of the intermittent, and eventually permanent, supersonic regions on the surface of cylinder along with the attendant surface shock waves up to the wake shock waves regime. The presence of supersonic regions prevents pressure fluctuations to be communicated upstream during the half period that the local flow is not supersonic, leading to reduced cylinder surface pressure perturbations. Thus, in the intermittent shock wave regime during the first half of the shedding cycle, the pressure fluctuations can move forward over one half of the surface and during the second they can move forward over the other half. The ability to prevent the fluctuations moving forward will be dependent on the size of the supersonic regions and so will increase with free stream Mach number as these regions become larger.

The cessation of pressure fluctuations over the windward side of the cylinder at Mach 0.7 indicates that localized areas of supersonic flow are, or are nearly, permanent. This is evidenced in Fig. 7. At Mach 0.6, Fig. $7 \mathrm{a}$, the time-averaged isentropic Mach number reaches a maximum just above unity at $\theta \approx 65^{\circ}$ and remains there over the range $65^{\circ} \leq \theta \leq 180^{\circ}$. This indicates that the intermittent shock waves are indeed present for only half of a vortex shedding cycle, allowing the movement upstream of pressure fluctuations during the half of the vortex shedding cycle that is not supersonic, resulting in sizable surface pressure fluctuations over this half cycle only. Note the maximum isentropic Mach number about the leeward side of the cylinder at Mach 0.6 shoots up to a very high indicated Mach number at some points. This is an artifact of the use of the pressure coefficient defined in Eq. 1, which is most suited to subsonic flows.

At Mach 0.7, Fig. 7b, the time-averaged isentropic Mach number accelerates to well above unity, indicating that the flow on both sides of the cylinder remains supersonic for a significant proportion of the time. While the flow on both sides of the cylinder is sonic there can be no, or in practice very little, communication of pressure fluctuations upstream so reducing the maximum and minimum levels of isentropic Mach number on the windward face. This is shown in Fig. $7 \mathrm{~b}$ by a reduction in width of the region delimited by the maximum and minimum isentropic Mach numbers with respect to Fig. 7a in the range $0^{\circ} \leq \theta \leq 60^{\circ}$.

The changes in flow features and vortex-shedding mechanisms can be seen from the changes of vortex shedding frequency with free stream velocity and Mach number, Fig. 8a. From Gerrard ${ }^{12}$, the shedding frequency may be viewed as the result of a balance between the size of the formation region and the thickness and state of the shear layers feeding into it. Figure 8a shows that the rate of increase in the fundamental vortex shedding frequency between Mach 0.6 and 0.8 remains linear. Above Mach 0.8 the rate of increase in vortex shedding frequency with free stream velocity increases and below Mach 0.6 initially decreases and then increases down to Mach 0.4. This seems to indicate that either the amount of fluid entrained into each vortex remains the same throughout the Mach 0.6 to 0.8 range or the rate of entrainment and the amount of fluid entrained changes in such a way as to create a linear increase in shedding frequency. Below Mach 0.6 and above Mach 0.8 either the amount of fluid entrained per vortex changes nonlinearly or the rate of entrainment changes nonlinearly, or both.

The apparently linear increase in shedding frequency between Mach 0.6 and 0.8 is interesting as over this Mach number range the flow regime changes twice, from intermittent shock waves to permanent shock waves and then to wake shock waves although the final change in regime has only just occurred at Mach 0.8. The formation of vortices, the subsequent increase, up until just over Mach 0.6, and then reduction in their strength and the increase in frequency at which they are shed is very complex and dependent on a number of variables.

A possible reason for the cessation of the linear increase in shedding frequency above Mach 0.8 can be found in the flow visualization of Dyment and Gryson ${ }^{13}$. From their flow visualization at Mach 0.8 and 0.9 the vortex formation region can be seen to lengthen by around two and half times. This suggests that, when the permanent shock wave regime first appears there is initially little change in the formation of the vortices. However as the flow moves further into the regime the differences between it and the intermittent shock wave 
a

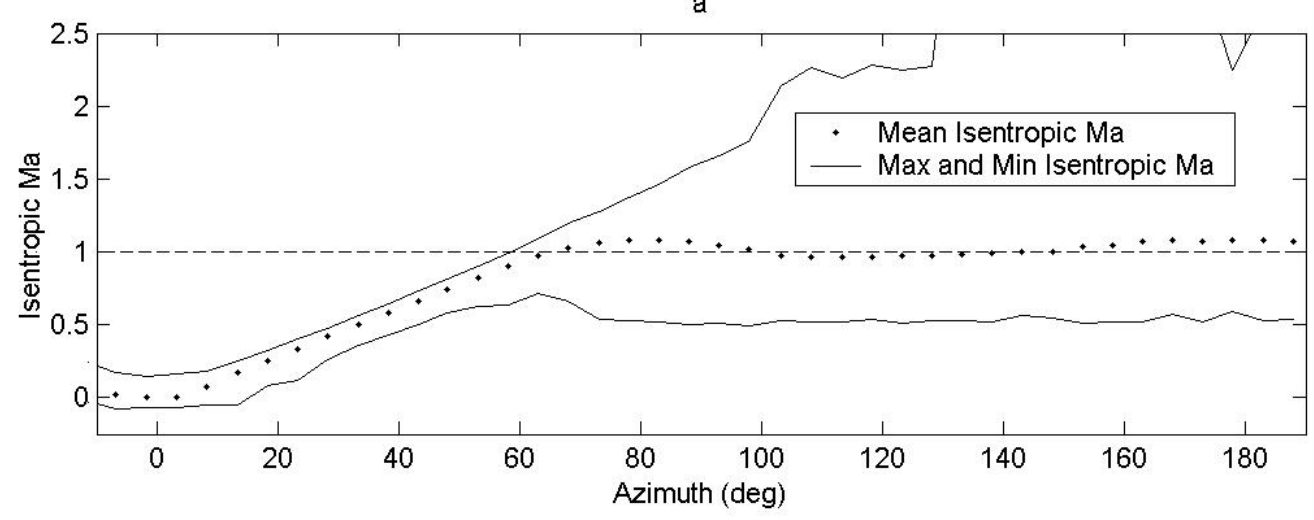

b

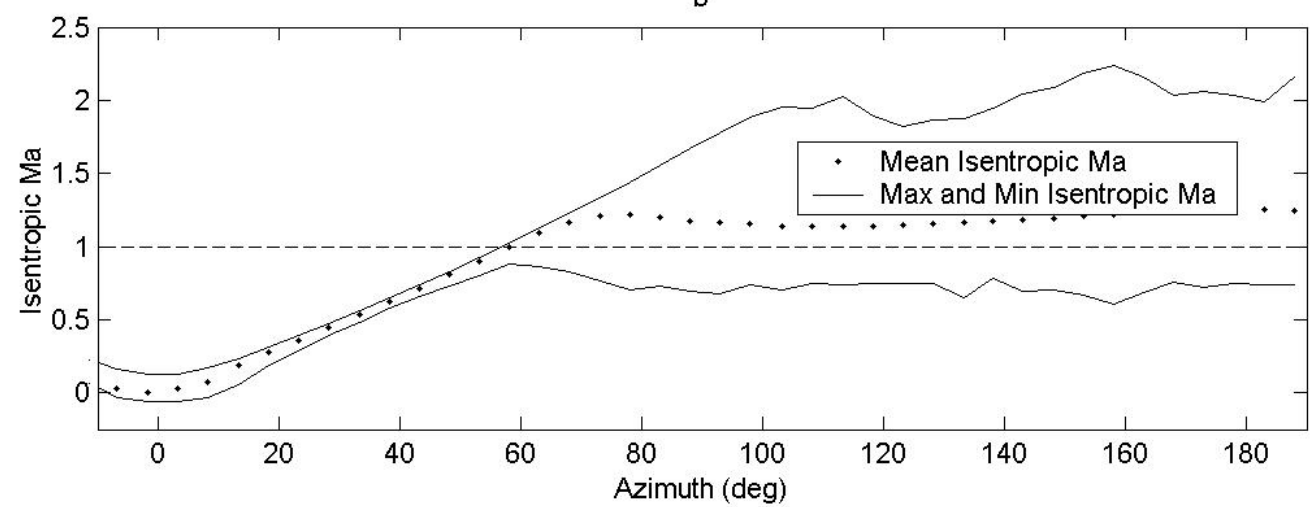

Figure 7. Isentropic Mach number distribution for 2000 series results

a) Mach 0.6, b) Mach 0.7

regime become more apparent as the flow features become stronger. It therefore seems reasonable to expect the nonlinear increase in shedding frequency to be related to the extension of the vortex formation region. Above Mach 0.9 the vortex formation becomes yet more complex as the shear layers start to converge, causing the vortex formation region to thin.

However, as noted below, at this Mach number it is impossible to determine Strouhal number from surface pressure measurements and so the changes here are unknown. The fundamental shedding frequencies were taken from frequency spectra of the data created using Matlab's psd function. A sample of these for each Mach number, each taken at an azimuth of around $85^{\circ}$, is given in Fig. 9 .

It is worth noting that there is very little difference in the vortex shedding frequencies found from the 2000 series and 2002 series of tests. For example, at Mach 0.6, where the biggest differences between the two series are found, the vortex shedding frequencies are $928 \mathrm{~Hz}$ and $944 \mathrm{~Hz}$ for the 2000 series and 2002 series results respectively, a difference of just $16 \mathrm{~Hz}$ or $1.7 \%$. At Mach 0.5 and 0.7 the differences between the two series are $18 \mathrm{~Hz}$ or $2.1 \%$ and $30 \mathrm{~Hz}$ or $2.7 \%$ respectively. This indicates that, while the difference in boundary layer state does have some effect on the vortex shedding frequency, this is very small.

It should be noted that at the Reynolds number used in these tests there should be no vortex shedding at all when the flow is fully subsonic as it will fall into the TrBL3 regime ${ }^{11}$. Indeed from a quick inspection of the frequency spectra, Fig. 9a, it appears that this is the case. However a closer inspection of the data reveals that there is a broad increase in the power spectrum magnitude in the region of around $550 \mathrm{~Hz}$ to $670 \mathrm{~Hz}$ at azimuths of around $80^{\circ}$ to $90^{\circ}$. Also a closer inspection of the time-resolved data, from around $80^{\circ}$ to $90^{\circ}$, indicates occasional sinusoidal fluctuations with wavelengths that match this broad frequency range. From this it is inferred that there is weak intermittent shedding of vortices at this Mach number. The Reynolds number used is very near the upper boundary of the TrBL2 flow regime, within which vortex shedding does occur. Furthermore the authors have ample evidence of vortex shedding occurring behind turbine blades under these conditions and much further into the transonic regime ${ }^{3}$.

The data were too sparse to fit a curve that allowed for the nonlinear behavior as the Mach number changed. However further insight can be gained into the behavior of the vortex shedding frequency through inspection of the non-dimensionalized vortex shedding frequency, the Strouhal number. When plotted against Mach number, as in Fig. $8 \mathrm{~b}$, the changes in Strouhal number confirm the indications given by the changes of shedding 

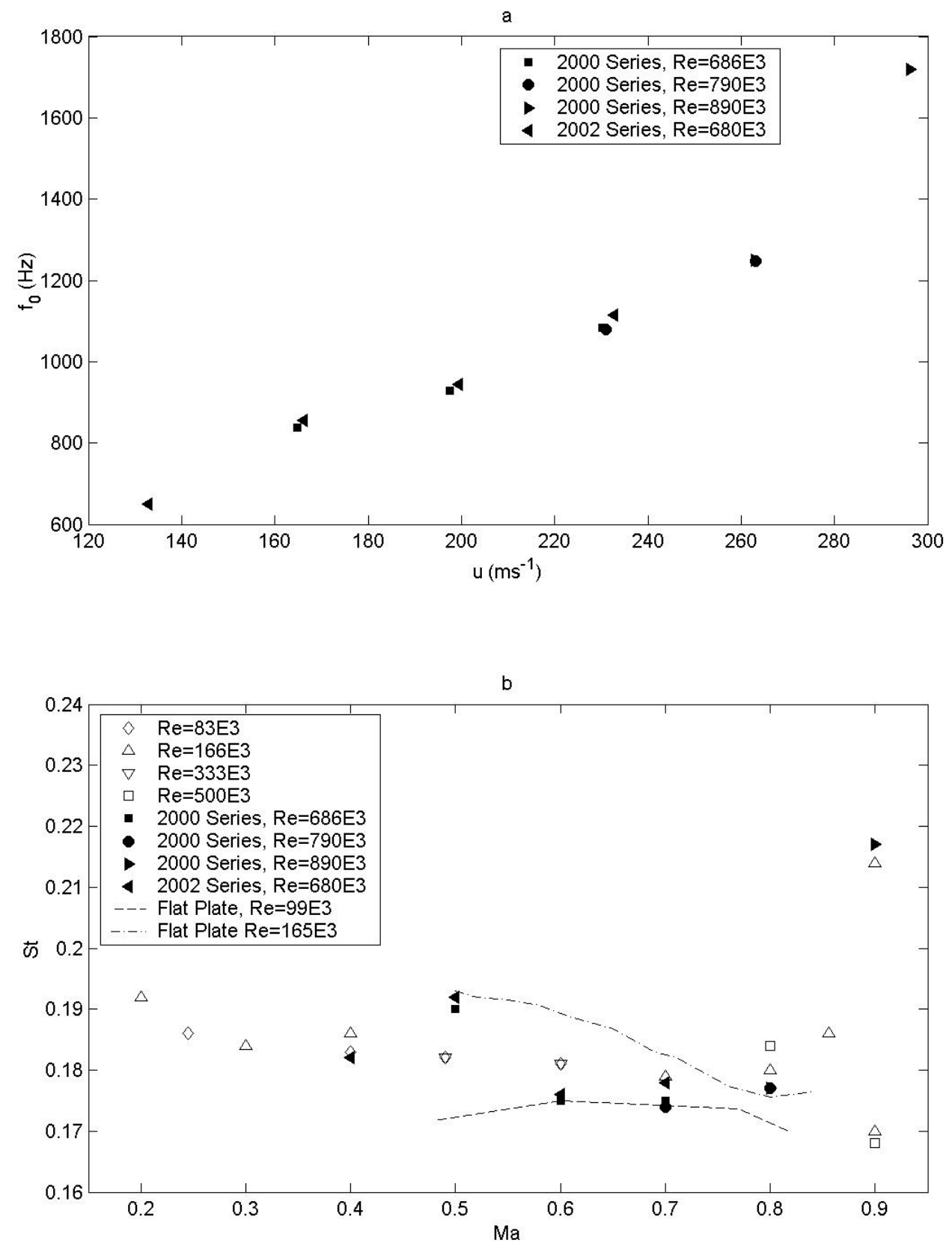

Figure 8, Vortex shedding frequency across experimental range,

a) Fundamental shedding frequency as a function of free stream velocity,

b) Strouhal number over the Mach number range.

$83 \times 10^{3}<\operatorname{Re}<500 \times 10^{3}$ (Open symbols): Murthy and Rose ${ }^{14}$

Flat plate: Heinemann, Lawaczeck and Bütefish ${ }^{15}$

frequency regarding the shedding mechanisms. This is because, as well as normalizing the data with respect to the cylinder diameter and free stream velocity, the Strouhal number is also the product of the first derivative of shedding frequency with respect to free stream velocity and the cylinder diameter. Thus any change in the rate of increase of the fundamental vortex shedding frequency with the free stream velocity shows up when Strouhal number is plotted against Mach number.

As the Mach number increases from 0.4 to 0.5 the Strouhal number, St, rises from 0.182 to 0.192 as the intermittent shock wave regime is established. St then falls off significantly from around 0.19 down to about 0.176 between 0.5 and 0.6 as the local supersonic regions become stronger. The Strouhal number then remains 

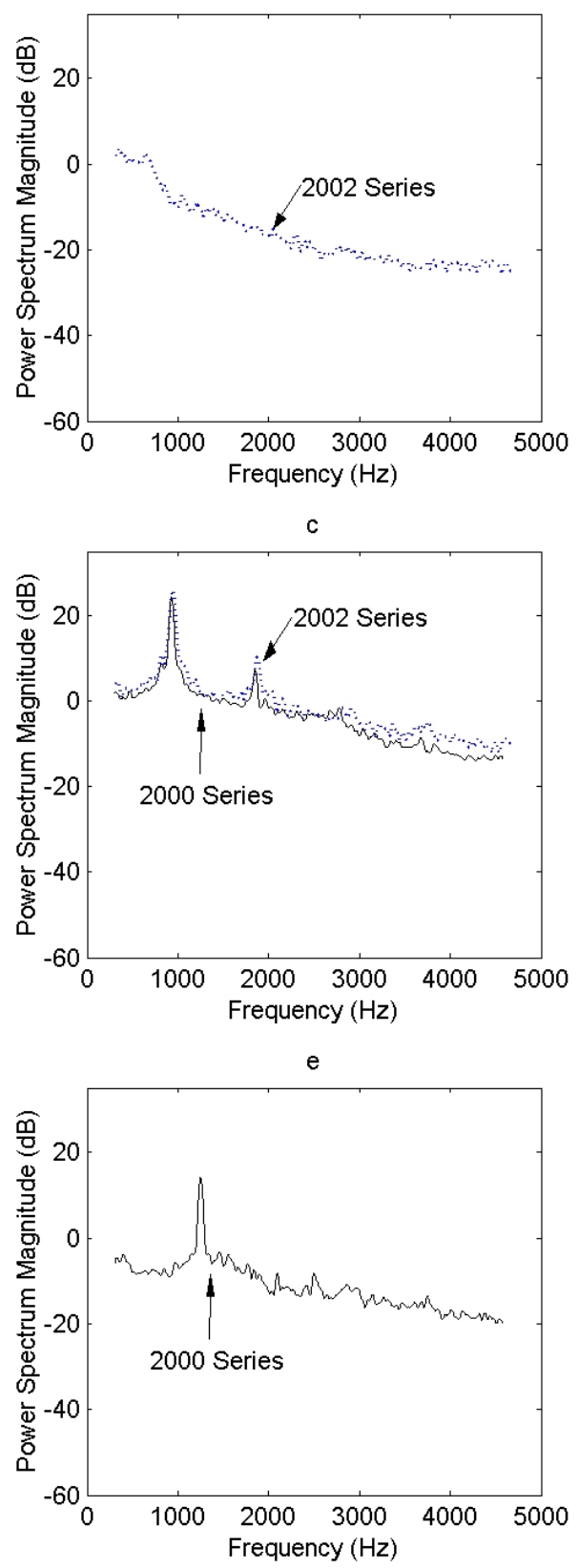

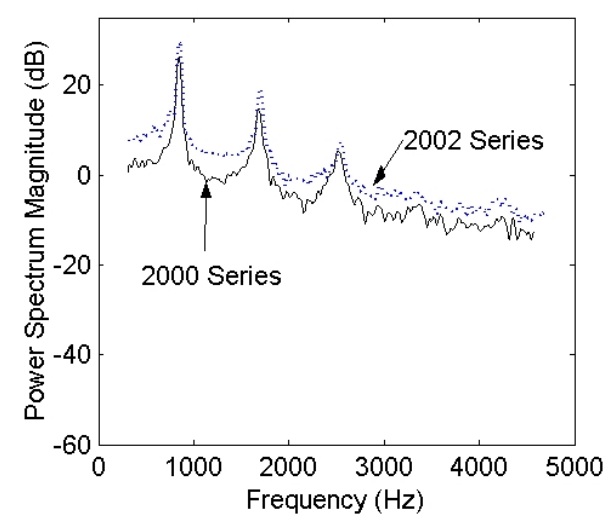

d

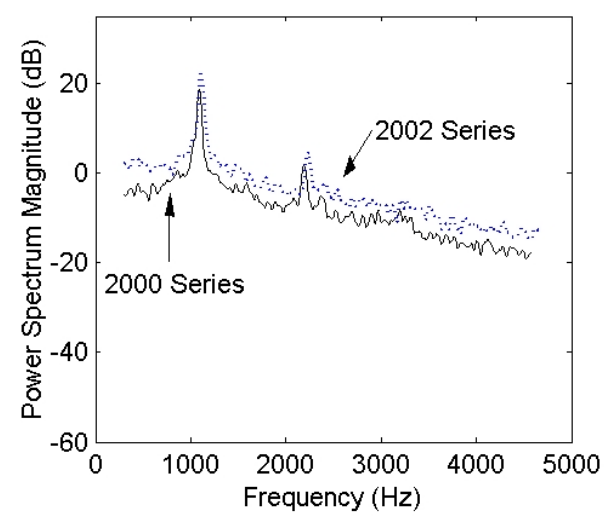

$f$

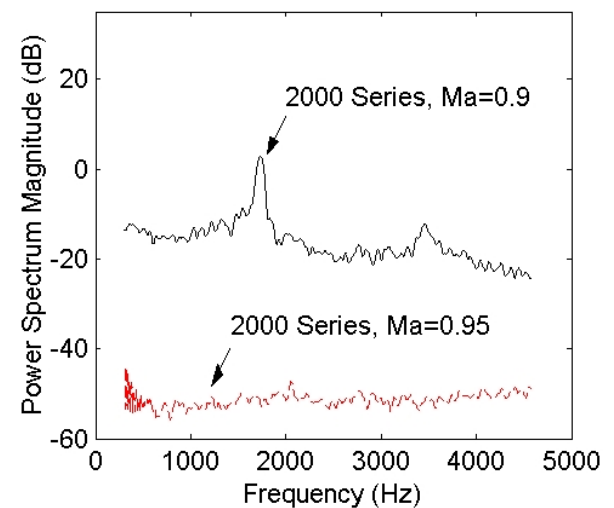

Figure 9. Frequency spectra of surface pressure results taken at azimuths of $83.21^{\circ}\left(2000\right.$ Series) and $84.21^{\circ}(2002$ series $)$

a) $\mathrm{Ma}=0.4$, b) $M a=0.5$, c) $M a=0.6$, d) $M a=0.7$, e) $M a=0.8$, f) $M a=0.9$ and 0.95

fairly constant through the intermittent shock wave and permanent shock wave regimes, confirming the linear increase of shedding frequency in this region, before increasing to 0.217 at Mach 0.9 as the wake shock wave regime strengthens.

These findings are supported by the circular cylinder results of Murthy and Rose ${ }^{14}$ and the flat plate work of Heinemann, Lawaczeck and Bütefish ${ }^{15}$ that are plotted alongside the results of the 2000 and 2002 series tests. The results from Murthy and Rose are broadly similar to those from these tests, although they do not show an increase in Strouhal number at Mach 0.5.

They did report that their findings at Mach 0.4 showed a broadband increase in the frequency spectra, indicating they had similar results to these but interpreted them differently. It is also interesting that at high 
Mach numbers Murthy and Rose found that the Strouhal number could either rise or fall. This would indicate that the vortex shedding at this Mach number is unstable, confirming the findings of Carscallen et $\mathrm{al}^{3}$.

The work of Heinemann, Lawaczeck and Bütefish is presented by way of comparison between the wake flow of flat plates (and by extension airfoils and the blades of turbines and compressors) and that of circular cylinders. While it is clear from the patterns formed by the different sets of data that the flow regimes for the two types of flow have different limits, this is to be expected given the low critical Mach number of circular cylinders. Regardless of the differences, the similarity between the two sets of data is encouraging.

Another point of interest is that from the data in Fig. $8 \mathrm{~b}$ there is little difference in the Strouhal number of data taken at different Reynolds numbers but the same Mach number. This indicates that once the flow around a circular cylinder has become critical, the Reynolds number ceases to be a major determining factor in the flow. It is also interesting to note that few Strouhal number data are given in the literature for Mach numbers above 0.9 . While Zdravkovich ${ }^{11}$ states that no vortex shedding is found above Mach 0.9 , the flow visualization ${ }^{13}$ that he uses at Mach 0.95 indicates that vortex shedding does occur up to that Mach number. However, as presumably with previous work, no periodic pressure fluctuation that would indicate the presence of vortex shedding was found on the surface of the cylinder at Mach 0.95 during the 2000 series of tests. This can be seen from the low RMS level and the narrow bars delimiting the maximum and minimum pressures in Fig. $2 \mathrm{f}$. However one should note that at Mach 0.95 a strong fishtail shock forms at the confluence of the free shear layers from which the vortices are formed and shed. It seems likely then that the supersonic region of flow responsible for this shock prevents pressure fluctuations from moving upstream to the cylinder surface where they could be detected.

In summary, it may be said that once the compressible flow has fully established itself, at around Mach 0.6, the base drag and drag coefficients begin to fall with increasing Mach number until the flow enters the wake shock wave regime. Once there, the drag and the base drag coefficients begin to rise again. In a similar vein the Strouhal number remains approximately constant from Mach 0.6 up to Mach 0.8 when the wake shock wave regime starts. It would seem then that the mechanism responsible for the formation of the vortex street remains the same through the intermittent shock wave and permanent shock wave regimes but, once the steadier near wake of the wake shock wave regime is established, the mechanism changes leading to increased base drag, despite an increase in pressure recovery.

The change in mechanism can be seen from the change in behavior of the time-resolved data both in the time and frequency domains. While the magnitude of the pressure coefficient fluctuations around the surface of the cylinder fall in the Mach number range 0.5 to 0.7 , the behavior remains the same. However at Mach 0.8 a sudden clearing up of the signal, accompanied by a significant drop in the size of the fluctuations and the introduction of a new behavior, described above under the description of the Mach 0.8 results, indicate a change in mechanism. This is accompanied by the disappearance of any pressure fluctuation on the windward face of the cylinder, i.e. before separation, and an increasingly sharp rise in the frequency content during and after separation, indicating a reduction in the movement of the separation shocks.

The results also showed that the state of the boundary layer before separation has little effect on the drag and base drag coefficients except at Mach 0.6. A possible explanation of this is the strength of the intermittent shock wave regime at Mach 0.6. Below this Mach number the regime is still establishing itself and above it, it is eradicated by the formation of the permanent shock wave regime. Mach 0.6 is where the difference in boundary layer state has the greatest effect on the flow immediately before, during and just after separation resulting in the greatest, and indeed only significant, difference drag measured from the two series of tests. It would seem, then, that the mechanism for the formation of the vortices has a very significant impact on the base drag of the cylinder.

\section{Conclusions}

Time-resolved pressure distributions have given information on the location and strength of surface pressure fluctuations on a circular cylinder in cross flow over the subsonic speed range. The corresponding drag and base drag coefficients have been provided. Data on Strouhal number variation over the subsonic speed range are also provided and are compared with measurements by other authors.

The cross flow about a circular cylinder changes significantly at higher subsonic velocities and into the transonic range. The flow regime changes a number of times, resulting in changes to the strength of the shed vortices, as evidenced by the reduction in size of the fluctuations on the leeward side of the cylinder as the Mach number is increased. The changing strength of the vortices can also be seen, at Mach numbers above 0.6, to reduce the base pressure coefficient, and hence the drag coefficient, up to a Mach number of 0.9 , where the onset of sonic flow increases the drag acting on the cylinder.

The time-resolved base pressure fluctuations at low Mach numbers are in agreement with the findings of Cicatelli and Sieverding ${ }^{8}$ with regard to the relative time spent in vortex formation and shedding. As the Mach number increases, the time spent in vortex formation tends to become equal to that spent in shedding. 
The paper has concentrated on providing some detailed data on base pressure rather than attempting to produce universal correlations. It was considered more important to provide detailed information and physical explanations of the various configuration changes where possible. This should be of assistance in eventually providing a more general model of the base pressure on a circular cylinder in cross flow over the subsonic range.

\section{Acknowledgments}

A research grant from Engineering and Physical Sciences Research Council is gratefully acknowledged. The authors are indebted to the National Research Council of Canada (NRC) for the use of the U-66 wind tunnel and workshop facilities without which this investigation would have been impossible. Particular NRC support was received from Drs. Hanff and Nguyen. Appreciation is expressed to Mr. P. Hunt and to the U-66 team for running the facility and providing help, support and advice.

\section{References}

${ }^{1}$ Williamson, R. G., and Moustapha, S. H., "Annular Cascade Testing of Turbine Nozzles at High Exit Mach Numbers," Journal of Fluids Engineering, Vol. 108, 1986, pp. 313-320.

${ }^{2}$ Denton, J. D., and Xu, L., "The Trailing Edge Loss of Transonic Turbine Blades,” Journal of Turbomachinery, Vol. 112, 1990, pp. 227-285.

${ }^{3}$ Carscallen, W. E., Currie, T. C., Hogg, S. I., and Gostelow, J. P., "Measurement and Computation of Energy Separation in the Vortical Wake Flow of a Turbine Nozzle Cascade," Journal of Turbomachinery, Vol. 121, No. 4, 1999, pp. 703-708.

${ }^{4}$ Deych, M. E., Fillipov, G. A., and Lazerev, L. Y. A., “Atlas of Axial Turbine Cascade Characteristics," Mash. Publishing House, Moscow, 1965.

${ }^{5}$ Craig, H. R. M. and Cox, H. J. A., "Performance Estimation of Axial Flow Turbines," Proc. I. Mech.E., Vol. 185, 1971, $32 / 71$

${ }^{6}$ Lawaczeck, O., and Heinemann. H. J., "von Kármán Streets in the Wakes of Subsonic and Transonic Cascades," AGARD CP 177, 1976.

${ }^{7}$ Hoerner, S. F., “Fluid Dynamic Drag," Published by the author, 1958.

${ }^{8}$ Cicatelli, G., and Sieverding, C. H., "The Effect of Vortex Shedding on The Unsteady Pressure Distribution Around the Trailing Edge of a Turbine Blade," ASME paper 96-GT-359, 1996,

${ }^{9}$ MacMartin, I. P. and Norbury, J. F. "The Aerodynamics of a Turbine Cascade with Supersonic Discharge and Trailing Edge Blowing,” ASME Paper 74-GT-120, 1974.

${ }^{10}$ Buttsworth, D. R., and Jones, T. V., "A Fast Response Total Temperature Probe for Unsteady Compressible Flows," Journal of Engineering for Gas Turbines and Power, Vol.120, 1998, pp. 694-701.

${ }^{11}$ Zdravkovich, M. M. "Flow around Circular Cylinders, Vol. 1: Fundamentals,” Oxford University Press, 1997.

${ }^{12}$ Gerrard, J. H., "The Mechanism of the Formation Region of Vortices behind Bluff Bodies," Journal of Fluid Mechanics, Vol. 29, part 2, 1967, pp. 259-272.

${ }^{13}$ Dyment, A., and Gryson, P., "Study of Turbulent Subcritical and Supercritical Flows by High-Speed Visualisation," AGARD CP 227, Paper No. 28, 1979.

${ }^{14}$ Murthy, V. S., and Rose, W. C., "Form Drag, Skin Friction, and Vortex Shedding Frequencies for Subsonic and Transonic Cross Flows on Circular Cylinder," Proceedings AIAA $10^{\text {th }}$ Fluid and Plasma Dynamics Conf., Albuquerque, 1977, pp. 677-687.

${ }^{15}$ Heinemann, H.-J., Lawaczeck, O., and Bütefisch, K. A., "von Kármán Wakes and Their Frequency Determination in the Wake of Profiles in Sub- and Transonic Regimes," IUTAM Symposium Transsonicum II, Springer, Berlin, 1975. 